\title{
Activation, attention, and short-term memory
}

\author{
NELSON COWAN \\ University of Missouri, Columbia, Missouri
}

\begin{abstract}
The premise of this paper is that the popular term "short-term memory" (STM) is vague because it is used to refer to either (1) the set of representations from long-term memory currently in a state of heightened activation or (2) the focus of attention or content of awareness. A more coherent conception of STM is hierarchical, with the focus of attention depicted as a subset of the activated portion of long-term memory. Research issues are discussed to illustrate that this simple conception of STM leads to testable predictions and useful lines of inquiry.
\end{abstract}

There is a simple, subjective description of one's shortterm memory (STM) and awareness with which few would disagree. A small set of ideas, objects, events, and so forth, fill one's current attention and awareness. A somewhat larger set is outside of this focus but nevertheless remains especially available should one want to shift attention to it. An example is something that was said about a minute ago. The present paper examines empirical evidence for this description and its relation to research on STM.

What are the most basic characteristics of STM, and how can they be examined? STM often seems needlessly confusing because two definitions have been used in the literature. First, some have considered it to be the set of elements from long-term memory that currently are in an activated state (e.g., the reverberatory circuits of Hebb, 1949). Second, others have considered it to be the items that are in the current focus of attention (e.g., the primary memory of James, 1890). At times, I even have seen these two definitions used as if they were interchangeable.

The two definitions cannot logically be interchangeable, if there is such a thing as the activation of memory outside of awareness. Such activation has been demonstrated by many recent studies, for example, those on the effects of masked primes on lexical access (for reviews, see Holender, 1986, and Merikle \& Reingold, 1990). Even if one does not believe the recent research on automatic semantic activation, the ubiquitous finding of priming effects for recently presented items suggests that at least previously attended items stay active in memory for a while after they leave awareness.

Here, then, is a maximally simple theoretical depiction of STM (see Figure 1), which has been distilled from $\mathrm{Co}-$ wan (1988). STM is represented as a nested subset of longterm memory. Specifically, the currently activated features comprise a subset of long-term memory, and the

A version of this paper was presented as part of a symposium entitled "Short-term Memory: Where Do We Stand?" at the 32nd Annual Meeting of the Psychonomic Society, San Francisco, November 1991. The work was supported by NIH Grant HD-21338. I thank Molly Potter and Richard Schweickert for their helpful comments. Address requests for reprints to the author at the Department of Psychology, 210 McAlester Hall, University of Missouri, Columbia, MO 65211. current focus of attention is in turn a subset of this activated memory.

Two immediate qualifications are in order. First, the boundaries shown in the figure could be fuzzy rather than discrete. Second, given the present limitations of the field of neurophysiology, the term "activation" does not refer to neural activity directly; indeed, for all we know, greater activation could correspond to more neural inhibition. Activation is better defined for our purposes more behaviorally (e.g., as the temporary state of memory representations that would allow these representations to have a priming effect on subsequent stimuli; cf. Cowan, 1988). In neural terms, the only expectation at present is that this activation would be represented as some particular spatiotemporal pattern of activity.

The model shown in Figure 1 obviously is very simple. One might wonder why many interesting distinctions were omitted, such as sensory versus abstract versus motor memory, verbal versus spatial memory, and so on. This omission is important and has to do with the intent of the model. The intent was not to develop a detailed, explicit model of how the processing system might work. The model is aimed instead at the necessary instead of the possible: the most basic memory faculties that must exist according to all available evidence. As far as we know at present, the various domains of STM such as sensory, abstract, and motor memory can be grouped together on the basis that they all may operate on the same dynamic principles. For example, representations of any one type may be susceptible to interference from subsequent stimuli with similar features. Stimuli with sensory features similar to the prior input would interfere with its sensory memory representations, stimuli evoking similar motor responses would interfere with its motor memory representations, and so on. The various types of activated memory also may have similar properties of memory decay. The key distinction, however, would be between activated memory features that are in the current focus of attention versus features outside of that focus (Cowan, 1988).

This theoretical conception of STM is similar in some ways to the working-memory model of Baddeley (1986). It differs from his model, though, in the present assump- 


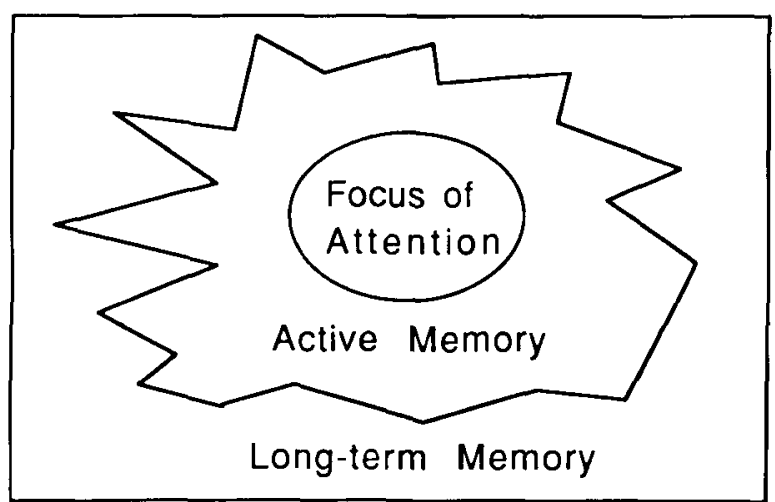

Figure 1. A schematic diagram of the memory system as discussed by Cowan (1988). STM has been defined either as the currently active portion of memory or as the current focus of attention.

tion that transient, activated memory of various types (sensory, phonological, semantic, and motor) may be instances of a common, general storage medium with many dynamic properties and principles that are common across features types, instead of separate, distinct modules such as Baddeley's visuospatial scratch pad and articulatory loop. It also differs because a wider variety of strategic mechanisms is considered. For example, although Baddeley's process of "covert rehearsal," which serves to reactivate items in memory, also can be described within the present conception (e.g., as a type of activated memory scheme that must be initiated within the focus of attention, although it might then carry on automatically), there also might be other memory reactivation mechanisms. We will see below that some of the work that Baddeley has attributed to covert rehearsal may be accomplished instead by high-speed search processes (Cowan, 1992).

The research that I will discuss begins to address three basic questions about the present, hierarchical view of STM. First, how long can stimuli be retained for immediate recollection when attention is directed toward as opposed to away from them? Second, what roles do activation and attention play in STM tasks? Third, how can we tell if the activated information in STM truly is based on information from long-term memory? These questions will be addressed in turn.

\section{Effects of Attention on Short-Term Retention}

Cowan, Lichty, and Grove (1990) investigated the relation between selective attention and memory retention in a task in which subjects had to read a novel, silently or by whispering, and were instructed to ignore syllables of speech presented through headphones. Occasionally, the subjects received a visual cue to stop reading and to identify the last syllable presented through headphones. This study was modeled after a previous one by Eriksen and Johnson (1964), except that we were looking at the delayed recognition of speech syllables rather than the delayed detection of tones. The subjects first received familiarization and practice with a set of spoken syllables presented through headphones. Then, in the test phase, they read a novel while the syllables were presented. Although syllables were presented at irregular intervals ranging from 1 to $13 \mathrm{sec}$, test trials occurred much less frequently, only 9 times per hour. In each test trial, the subject was to put down the novel and identify the last syllable presented by circling the correct choice out of 9 possible syllables depicted on an answer sheet. This last syllable occurred 1,5 , or $10 \mathrm{sec}$ before the test cue, so memory decay functions could be plotted from the data. The delay period always was quiet because there were no intervening syllables, and subjects either read silently or whispered the reading.

After identifying the last spoken syllable, the subject was to write a sentence about what was going on most recently within the reading. Finally, at the end of the session, there was a reading comprehension test. To keep subjects honest, they were told about the final reading test at the beginning of the experimental session. There were several control experiments that demonstrated that the auditory stimuli did not detract from the subjects' reading ability.

The nine test syllables were [bi], [bI], [bE], [di], [dI], [dE], [gi], [gI], and [gE]. Because they consist of the three consonant phones [b], [d], and [g] crossed with the three vowel phones [i], [I], and [E] (as in bean, bin, and Ben, respectively), we could score performance on consonants and vowels separately. This is of some value because the three vowels are more discriminable than the three consonants; therefore, performance can be viewed at two levels of difficulty. The response sheet looked like a $3 \times 3$ matrix.

In the most informative experiment in the set (Cowan et al., 1990, Experiment 4), the subjects whispered the reading. In the practice session in which subjects listened to the syllables and did not read, identification was over 90\% correct for both consonants and vowels. However, memory for syllables that were to be ignored at the time of presentation dropped off dramatically as the postsyllabic delay increased from 1 to $10 \mathrm{sec}$. Similar decay functions were obtained for consonants and vowels, although at a much higher overall level for the vowels.

In this experiment, we also were able to test for subtle shifts of attention away from the reading inasmuch as the subjects had whispered the reading instead of reading silently. We recorded the whispering and were able to examine it for pauses. If a subject did not whisper at all for either $1 \mathrm{sec}$ before or $1 \mathrm{sec}$ after the onset of the target syllable, we reasoned that a shift of attention away from the reading and toward the headphone channel could have occurred. Measured in this way, possible attention shifts occurred on about $20 \%$ of the trials. On those trials, there was not much difference from the previous result for memory of the easier, vowel phonemes, but there was a dramatic difference for the more difficult, consonant phonemes. In fact, for the shorter delay intervals, this subtle shift of attention improved performance by about $20 \%$. The benefit of attention diminished at longer delays, as 
the subjects usually turned their attention back to the reading.

What happens when attention is more consistently divided between the two channels? To examine this in another experiment (Cowan et al., 1990, Experiment 3), subjects not only read the novel, they also were to press a button every time that a [dI] occurred through headphones. The syllabic-recognition test trials used in the previous experiment also were administered in this one. The subjects were able to detect about $60 \%$ of the [dI] syllables while reading, with a very low false-alarm rate. However, the memory performance under these divided attention circumstances was very different. There was very little forgetting of the target syllable, even at the 10-sec delay.

The results of these experiments suggest, first, that there is considerable memory for spoken stimuli that are not fully attended at the time of presentation, although this memory decays rapidly. Second, subtle shifts of attention toward particular stimuli can be shown to markedly improve memory for these stimuli.

\section{Activation and Attention in STM Tasks}

The second research question concerns the role that activation and attention play in more typical STM tasks. The kind of task that I have examined, to begin to look at this question, is the common one in which a list of words is presented and the subject is simply to repeat the list immediately after its presentation. One possible role of activation can be inferred from the assumption (Baddeley, 1986; Cowan, 1988) that the items in a verbal list are represented in the form of a temporarily activated sequence of units. These units decay after activation unless there is some process that reactivates them.

One prediction from this conception of short-term recall is as follows. While a subject is receiving a list of items and is not currently responding to the list, rehearsal processes may reactivate items. However, whenever the subject is busy verbally repeating an item, it presumably is impossible to rehearse the other items at the same time. For this reason, activated memory should decay, at least at certain times, within the response period.

This prediction was examined by Cowan et al. (1992). The stimulus words were inspired by some that Baddeley, Thomson, and Buchanan (1975) used. The two word sets had identical numbers of phonemes and syllables. However, with such stimuli, it still takes subjects longer to pronounce words in the set marked "long" than in the set marked "short." Therefore, the processing of words in the long set should allow more memory decay to occur. Baddeley et al. found that memory performance was better for short sets than for longer sets, and we replicated this finding.

Baddeley et al.'s (1975) word-length effect could occur for at least two different reasons, however, corresponding to the two paths of articulation in the STM model: covert rehearsal and overt pronunciation. The reason favored by Baddeley et al. was that the longer words slow down the rehearsal process. This may be true, but we were interested in the effects of word length on the decay of memory during the subject's overt verbal response to the list.

To examine the effects of word length on overt recall processes, we used mixed lists. The length of words in the first and second halves of each list was varied independently. Therefore, there were four types of lists: shortshort, short-long, long-short, and long-long, according to the length of words in each half-list. (Stated more precisely, inasmuch as five-word lists were used, the length of the middle word was counterbalanced across trials in each of the length combinations.) This procedure was similar to an experiment conducted by Watkins (1977), except that he manipulated word frequency rather than word length and used a span task, which could not yield the serial position information that we found to be especially informative. Subjects received a printed list at the rate of $2 \mathrm{sec} /$ word. In the most informative experiment, there was a printed cue following the last word in the list that told the subjects to recall the words in either forward or backward order.

We reasoned that, if there were an effect of word length during the repetition of the list, the specific effects of word length would depend on the order of recall. The words that are repeated first delay the output of the remaining words in the list, and the lengths of those words repeated first should have a large effect on recall. In contrast, the words that are repeated last do not delay the output of any other words, and they therefore should have much less effect.

The results were very clearly as predicted. When words were to be recalled in the forward order, there was a significant effect of the length of words in the first half of the list, and this effect extended across the list. However, there was no effect of word length in the second half of the list. In the backward recall order, though, the results were just the opposite. There, it was the length of words in the second half of the list that made a difference for recall throughout the list, not the length of words in the first half of the list. In other words, it was always the length of just the words in the part of the list to be repeated first that made a significant difference. This pattern of results is reproduced in Figure 2. It supports the idea that during the time when a subject repeats any one word in the list, the memory for the other words in the list can decay from activation.

This finding is only a start toward answering the question of what roles activation and attention play in STM tasks. There are two alternative, slightly more detailed descriptions of what might happen as subjects repeat words in such tasks. The first possibility is that memory activation decays steadily while the subject repeats words. According to this account, the subject would have to finish his or her recall before too much memory decay takes place. Across many memory span studies with a variety of subject groups and stimulus conditions, it has been observed that people can remember about as much as they 

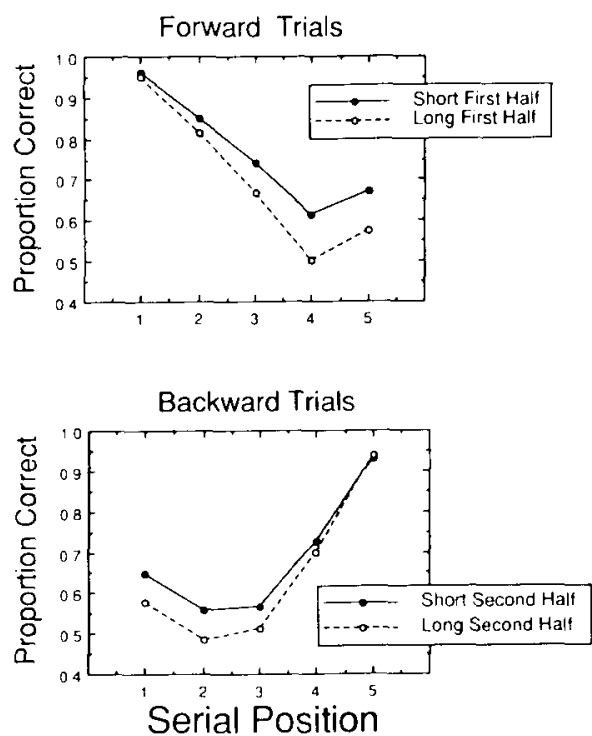

Figure 2. Results of Experiment 3 of Cowan et al. (1992). Top panel: Forward recall as a function of serial position for lists with a short versus long first half (collapsed across the second-half word length, which was not significant). Bottom panel: Backward recall as a function of serial position for lists with a short versus long second half (collapsed across the first-half word length, which was not significant). Notice that it was always the length of words to be pronounced first that made a difference for recall. Reprinted from $\mathrm{Co}$ wan et al. (1992) with the permission of Academic Press.

can say in about $2 \mathrm{sec}$ (for a review, see Baddeley, 1986). Therefore, some researchers (Schweickert \& Boruff, 1986; Stigler, Lee, \& Stevenson, 1986) simply have assumed that a version of this first mechanism is correct, with an effective memory decay period of about $2 \mathrm{sec}$. Their hypothesis is that the period of repetition of a spanlength list is limited to little more than $2 \mathrm{sec}$.

Most of the studies on the relation of speech rate to memory span have based their findings on the rate of speeded pronunciation in special tasks in which the subject is to repeat just a few words as quickly as possible. They have not focused on the actual duration of the subject's repetition of the lists to be recalled. However, the 2-sec-recall-period hypothesis mentioned above would seem to require that type of data. Stigler et al. (1986) did examine the duration of spoken recall responses and found a mean for adults' overt pronunciation of span-length word lists of under $3 \mathrm{sec}$, both in English- and in Chinesespeaking subjects, even though there was a higher span length in the latter.

Despite the finding of Stigler et al. (1986), an alternative, more complex account of processing during the recall phase cannot be ruled out. While a subject devotes attention to speaking a particular word, there would indeed be some decay of the activation of other words, but during pauses between words, some reactivation of memory would take place as the subject scans, rehearses, or in some other way processes some of the items that are still to be recalled. This scanning might or might not be conducted for the specific purpose of reactivating items: all that is necessary is the assumption that items processed during interword pauses are in fact reactivated in memory. This account could be correct, despite the findings of Stigler et al., because it might be that the reactivation periods in their adult subjects were rapid enough so that the recall period was not extended much beyond the expected 2-sec limit.

Even without making a commitment about exactly what process takes place during the pauses between words during recall, the hypothesis of a decay-and-reactivation cycle, with decay during word pronunciations and reactivation of some words during interword pauses, leads to a distinct prediction. The duration of span-length spoken recall need not correlate with memory span. Subjects who can remember more might do so by extending the total duration of memory activation, and they might achieve that by engaging in more rapid or efficient covert processing in the pauses between words.

To distinguish between the two accounts of STM recall (monotonic decay vs. decay-and-reactivation cycles), Cowan (1992) examined some tape-recorded protocols for memory span in forty-four 4-year-old children by using an acoustic waveform editor (MacRecorder Soundedit) to measure the timing of recall. The hope was that processes causing reactivation would take place more slowly, and thus be more easily detectable, in these subjects than in adults. The subjects' memory spans varied from two to five items. The duration of recall was measured in a number of slightly different ways, but the result was the same for each method. The duration of recall certainly was not limited to $2 \mathrm{sec}$ or to any other fixed period. Instead, there was a linear relation between an individual's memory span and the duration of that individual's recall for span-length lists. In fact, the children with the highest spans produced responses that lasted for almost $5 \mathrm{sec}$. Also, in contrast to the finding in the literature (e.g., Baddeley et al., 1975) of a strong correlation between memory span and the rate of pronunciation in speeded pronunciation tasks, no relation between an individual's memory span and speech rate in spoken recall was observed.

One way to explain the strong relation between span and the duration of recall in span-length lists while defending the monotonic decay theory would be to assume that the duration of decay varies markedly among subjects (e.g., from about 2 to $5 \mathrm{sec}$ in the 4-year-old subjects). However, that type of account could not explain why people can recall about as much as they can say in a speeded task in about $2 \mathrm{sec}$, regardless of memory level. A better account of all of the data is the decay-and-reactivation account. It would suggest that individual differences in span result from differences in the speed and/or efficiency with which subjects can retrieve and reactivate items in the pauses between words in the response.

Additional findings of Cowan (1992) yield further information about what may be going on in recall. Although children of different spans pronounced their recall at comparable rates within their respective span-length lists 
(about 1.2 words $/ \mathrm{sec}$ ), they pronounced lists of length span -1 at a faster rate (about 1.4 words $/ \mathrm{sec}$ ), and lists of length span -2 at a still faster rate (about 1.6 words/ sec). Moreover, this effect of list length relative to span on pronunciation rate was localized entirely in the pauses between words, with no effect of list length on the duration of words in the response. These findings are entirely parallel to what has been obtained previously by Sternberg and his colleagues (Sternberg, Monsell, Knoll, \& Wright, 1978; Sternberg, Wright, Knoll, \& Monsell, 1980 ) in a different procedure, in which subjects received a ready signal on each trial and then pronounced a subspan-length list as quickly as possible. Sternberg and his colleagues have argued on the basis of many types of converging evidence for a model in which subjects rapidly scan the list during pauses in the response, in search of the item with the correct serial position marker to be pronounced next. It seems likely that this scanning process, or whatever search process actually accounts for the timing of speech in Sternberg and his colleagues' procedure, will end up accounting for the timing of spoken recall in span tasks as well. This is encouraging, given that there is a known relationship between memory search rate and memory span (Cavanagh, 1972). It strengthens the hypothesis that individual differences in memory span might be accounted for by differences in the efficiency of memory search taking place during pauses in the response.

In one way, this result is reminiscent of the finding of Cowan et al. (1990) regarding memory for ignored syllables of speech. In both cases, it appears that there is a decay of memory activation over time but that there are subtle but frequent shifts of attention that initiate processes resulting in reactivation. In ordinary STM tasks, it often may be difficult to observe the decay of activation precisely because subjects are frequently shifting attention between items in order to postpone memory decay, even within a single response sequence. This activation-attention interaction should be a key topic for additional investigation.

\section{Concept of Short-Term Activation of Long-Term Memory}

The third general research question posed at the beginning of this paper was whether activated memory necessarily is drawn from long-term memory, as was proposed. (An alternative possibility is that STM is a completely separate resource or structure, as proposed for example by Broadbent, 1984, and Shallice \& Warrington, 1970.) A number of recent studies of other investigators are relevant here. First, Charles Hulme and his colleagues (Hulme, Maughan, \& Brown, 1991) did a study of speeded pronunciation and memory span in adults, using both English words and nonsense words as stimuli. The usual linear relation between speech rate and memory span was obtained, but with a much higher intercept for words than for nonwords. This illustrates that one's long-term lexical familiarity with the material to be activated makes a big difference in STM tasks.

Second, some new research by Cantor and Engle (1992) shows a clear inverse relation between an individual's working memory span and the magnitude of his or her propositional fan effect. Of course, fan effects (in which the mean time to recognize that two items have appeared together in an experiment is positively related to how many other items have been paired with either of the two items in question) have been used to demonstrate limitations in the spread of semantic activation through the propositional network in long-term memory (Anderson, 1974). The implication of Cantor and Engle's study is that working memory span also may depend on the extent of network activation.

Nairne's (1992) research on the long-term serial recall of verbal lists also is relevant. Previous studies on immediate recall (e.g., Lee \& Estes, 1981) show a very systematic set of order errors. Nairne's findings are similar but concern delayed recall over minutes or even hours. One explanation of this finding is that STM sets up a pattern of activation that becomes etched into long-term memory also, in a Hebbian (see Hebb, 1949) manner.

Across all of these recent studies, it seems fair to say that long-term memory is both the source and the beneficiary of the activation pattern in STM.

\section{Concluding Remarks}

Theoretically, STM serves as the interface between everything we know and everything we perceive or do. Yet, there is quite a gap between this central theoretical role of STM and the much more confined scope revealed by the conventional procedures for examining STM (e.g., digit span). One way to bridge this gap is to expand the arsenal of procedures, but another way is to work harder to obtain a better understanding of the role of theoretical mechanisms in the conventional tasks. However, it is my belief that there has been considerable confusion in the use of terms and that this has at times gotten in the way of a theoretical understanding of the mechanisms operating in STM tasks.

A simple suggestion of Cowan (1988) is that STM is a hierarchical construct, with one component being the currently activated portion of the memory system and another component being the subset of this activated memory that currently is in the focus of attention. However, this conception of STM serves only as a beginning, not an ending, point. I believe that it is a good beginning because it permits a search for general principles and deliberately avoids making further subdivisions of STM for which the processes have not been proved to operate in fundamentally different ways. Even when differences are observed, it is important to consider if they arise specifically from different STM modules or from differences in contributing perceptual processes. For example, the well-known auditory modality superiority in list recall 
tasks does not imply that the decay period is longer in audition than in vision, since various data suggest that it probably results instead from the basically more temporal organization of audition as opposed to the more spatial organization of vision (Cowan, 1988; Penney, 1989).

From this beginning, it was shown that various lines of research can make progress by focusing on the details of specific mechanisms of activation and attention in STM tasks. Many fundamental questions remain to be addressed. For example, how can the amount of activation and attention be measured? Are the boundaries between these subsets of memory discrete or continuous? Is the focus of attention simply the subset of memory with the highest level of activation, or does it differ in a qualitative manner from memory outside of the focus? Although the present theoretical approach is simple and perhaps intuitively obvious, it still can lead to interesting empirical ramifications that are not so obvious and that clearly warrant further research.

\section{REFERENCES}

ANDERSON, J. R. (1974). Retrieval of propositional information from long-term memory. Cognitive Psychology, 6, 451-474.

BadDELEY, A. D. (1986). Working memory. Oxford, UK: Clarendon.

Baddeley, A. D., Thomson, N., \& Buchanan, M. (1975). Word length and the structure of short-term memory. Journal of Verbal Learning \& Verbal Behavior, 14, 575-589.

Broadbent, D. E. (1984). The Maltese cross: A new simplistic model for memory. Behavioral \& Brain Sciences, 7, 55-94.

CANTOR, J., \& ENGLE, R. (1992, November). A test of an activation notion of working memory. Paper presented at the annual meeting of the Psychonomic Society, St. Louis.

Cavanagh, J. P. (1972). Relation between the immediate memory span and the memory search rate. Psychological Review, 79, 525-530.

Cowan, N. (1988). Evolving conceptions of memory storage, selective attention, and their mutual constraints within the human information processing system. Psychological Bulletin, 104, 163-191.

Cowan, N. (1992). Verbal memory span and the timing of spoken recall. Journal of Memory \& Language, 31, 668-684.

Cowan, N., DaY, L., Saults, J. S., Keller, T. A., Johnson, T., \& FLORES, L. (1992). The role of verbal output time in the effects of word length on immediate memory. Journal of Memory \& Language, 31, 1-17.

Cowan, N., Lichty, W., \& Grove, T. R. (1990). Properties of mem- ory for unattended spoken syllables. Journal of Experimental Psychology: Learning, Memory, \& Cognition, 16, 258-269.

Eriksen, C. W., Johnson, H. J. (1964). Storage and decay characteristics of nonattended auditory stimuli. Joumal of Experimental Psychology, 68, 28-36.

НЕвв, D. O. (1949). The organization of behavior: A neuropsychological theory. New York: Wiley.

HolendER, D. (1986). Semantic activation without conscious identification in dichotic listening, parafoveal vision, and visual masking: A survey and appraisal. Behavioral \& Brain Sciences, 9, 1-66.

Hulme, C., Maughan, S., \& Brown, G. D. A. (1991). Memory for familiar and unfamiliar words: Evidence for a long-term memory contribution to short-term memory span. Journal of Memory \& Language, 30, 685-701.

JAMES, W. (1890). The principles of psychology. New York: Henry Holt. LEE, C. L., \& EsTES, W. K. (1981). Item and order information in shortterm memory: Evidence for multilevel perturbation processes. Journal of Experimental Psychology: Human Learning \& Memory, 7 , 149-169.

MerikLe, P. M., \& Reingold, E. M. (1990). Recognition and lexical decision without detection: Unconscious perception? Journal of $E x-$ perimental Psychology: Human Perception \& Performance, 16, 574583.

NAIRNE, J. S. (1992). The loss of positional certainty in long-term memory. Psychological Science, 3, 199-202.

Penney, C. G. (1989). Modality effects and the structure of short-term verbal memory. Memory \& Cognition, 17, 398-422.

SCHWEICKERT, R., \& BoRUFF, B. (1986). Short-term memory capacity: Magic number of magic spell? Journal of Experimental Psychology: Learning, Memory, \& Cognition, 12, 419-425.

Shallice, T., \& Warrington, E. K. (1970). Independent functioning of verbal memory stores: A neuropsychological study. Quarterly Journal of Experimental Psychology, 22, 261-273.

Sternberg, S., Monsell, S., KNoll, R. L., \& Wright, C. E. (1978). The latency and duration of rapid movement sequences: Comparisons of speech and typewriting. In G. E. Stelmach (Ed.), Information processing in motor control and learning (pp. 117-152). New York: Academic Press.

Sternberg, S., Wright, C. E., Knoll, R. L., \& Monsell, S. (1980). Motor programs in rapid speech: Additional evidence. In R. A. Cole (Ed.), Perception and production of fluent speech (pp. 507-534). Hillsdale, NJ: Erlbaum.

Stigler, J. W., Lee, S.-Y., \& Stevenson, H. W. (1986). Digit memory in Chinese and English: Evidence for a temporally limited store. Cognition, 23, 1-20.

WATKINS, M. J. (1977). The intricacy of memory span. Memory \& Cognition, $5,529-534$.

(Manuscript received March 17, 1992; revision accepted for publication June 24, 1992.) 